\title{
Reflexiones de enfermeras egresadas de la Universidad de La Laguna (España) sobre la comisión de un error en el contexto laboral
}

\author{
Graduated Nurses reflections from the University of La Laguna \\ (Spain) about making a mistake in job's context
}

\section{Reflexões enfermeiros graduados da Universidade de La Laguna} (Espanha) a cometer um erro no contexto do emprego

\author{
Cristo Manuel Marrero González ${ }^{1}$ Alfonso Miguel García Hernández² \\ ${ }^{1}$ Enfermero en el Servicio Canario de la salud. Área de salud de Tenerife (España). Doctorando en la Universidad de La Laguna. \\ ${ }^{2}$ Licenciado en enfermería. Doctor en Antropología Social y Cultural por la Universidad de La Laguna. Profesor titular de la \\ Universidad de La Laguna (España). \\ Cómo citar este artículo en edición digital: Marrero González, C., García Hernández, A. (2017). \\ Reflexiones de enfermeras egresadas de la Universidad de La Laguna. Cultura de los Cuidados (Edición digital), 21(48). \\ Recuperado de http://dx.doi.org/10.14198/cuid.2017.48.06 \\ Correspondencia: Calle Infante Juan Manuel 16, 38010. Santa Cruz de Tenerife. Tenerife. España. \\ Correo electrónico: cmmarrerogonzalez@gmail.com \\ Recibido: 14/02/2017; Aceptado: 20/05/2017
}

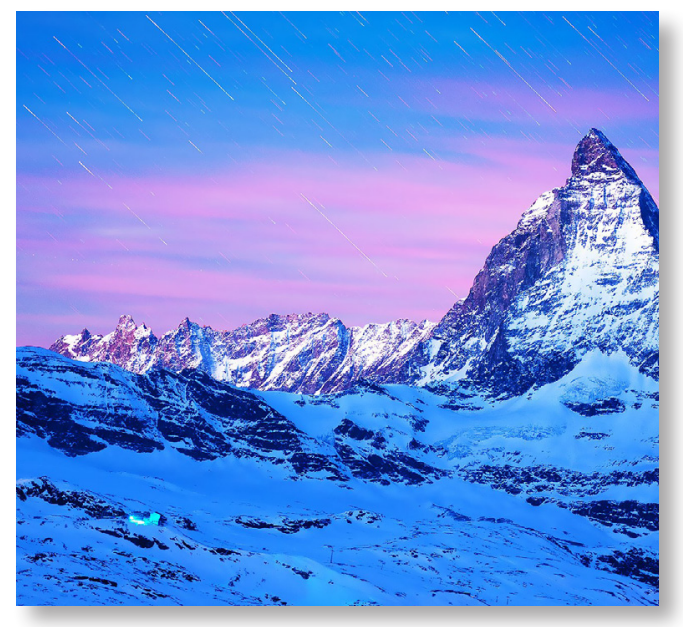

ABSTRACT

Objective: To deepen the experience of the graduated nurses from the University of La Laguna between 2009 and 2014 regarding responsibility, insecurity and fear of error in the labor context of Tenerife (Spain).

Methodology: a qualitative research is carried out with hermeneutical phenomenological approach through discourse analysis, having used as a data collection tool the semi-structured interview. Nineteen nurses participate, randomly selected and for convenience.

Results: the main category is "do". It is established as the main category of the study. Of the same the following subcategories are broken down: "to make a mistake"; "Different mistakes"; "Medication errors"; "Work overload errors; "To cause death"; "Recognize mistakes and learn from them" and "act wisely".

Discussion and conclusions: The meanings of the experiences are centered on making a possible mistake and its consequences. The experiences referred by the nurse with respect to responsibility are attributed fundamentally to being able to perform an adequate praxis in their work, being aware that they deal with people for it is an experience that brings much meaning to the nurse once it begins to work.

Keywords: Novice nurse, job; responsability, mistake. 


\section{RESUMEN}

Objetivo: Profundizar en las vivencias de las enfermeras egresadas de la Universidad de La Laguna entre 2009 y 2014 con respecto a la responsabilidad, la inseguridad y el temor al error en el contexto laboral de Tenerife (España).

Metodología: se realiza una investigación cualitativa con enfoque fenomenológico hermenéutico mediante el análisis del discurso, habiendo utilizado como herramienta de recogida de datos la entrevista semiestructurada. Participan 19 enfermeras, elegidas aleatoriamente y por conveniencia.

Resultados: se establece como principal categoría del estudio la denominada "hacer". De la misma se desglosan las siguientes subcategorías: "cometer un error"; "diferentes errores"; "errores en la medicación"; "errores por sobrecarga de trabajo; "provocar la muerte"; "reconocer los errores y aprender de los mismos" y "actuar con prudencia”.

Discusión y conclusiones: Los significados de las vivencias están centrados en cometer un posible error y en sus consecuencias. Las vivencias referidas por la enfermera con respecto a la responsabilidad se atribuyen fundamentalmente a poder realizar una adecuada praxis en su trabajo, al ser conscientes de que tratan con personas por ello es una vivencia que aporta mucho significado para la enfermera una vez que comienza a trabajar.

Palabras clave: Enfermera novel, puesto de trabajo, responsabilidad, error.

\section{RESUMO}

Objetivo: aprofundar as experiências de enfermeiros diplomados da Universidade de La Laguna, entre 2009 e 2014 no que diz respeito à responsabilidade, insegurança e medo de errar no contexto do emprego de Tenerife (Espanha).
Metodologia: pesquisa qualitativa é feita com abordagem fenomenológica hermenêutica através da análise do discurso, tendo utilizado como uma ferramenta para a entrevista semi-estruturado de coleta de dados. Envolvidos 19 enfermeiros, escolhidos aleatoriamente por conveniência.

Resultados: categoria principal está definido para estudar o chamado "fazer". Da mesma forma as seguintes subcategorias estão quebrados: "cometer um erro"; "Diferentes erros"; "Os erros de medicação"; "erros por excesso de trabalho; "Causar a morte"; "Reconhecer erros e aprender com eles" e "agir com prudência".

Discussão e conclusões: Os significados das experiências estão focados em fazer um possível erro e suas consequências. As experiências relatadas pela enfermeira sobre a responsabilidade é atribuída principalmente para permitir a prática apropriada em seu trabalho, esteja ciente de que lidar com as pessoas, por isso é uma experiência que traz um monte de significado para a enfermeira, uma vez que começa a trabalhar

Palavras chave: Enfermeira principiante, trabalho, responsabilidade, erro.

\section{INTRODUCCIÓN}

Las enfermeras recién egresadas, ven como desafíos que les preocupan aspectos relacionados con la inseguridad, la responsabilidad, la preparación, el entrenamiento, los conocimientos y la seguridad. Le dan muchísima importancia a sus primeras experiencias en los primeros años como profesionales. La transición es de por síun reto, máxime porque las enfermeras noveles se centran en las tareas y exigencias organizativas de la institución de trabajo, mientras sus capacidades se ven limitadas por su ansiedad, 
por la falta de experiencia y por la limitación de desarrollar juicios clínicos. Por esta razón, las enfermeras sienten frustración porque no pueden cumplir con sus expectativas como profesionales competentes. Los principales errores que comenten las nuevas enfermeras se relacionan con la administración de fármacos, de ahí que se sientan preocupadas por la seguridad del paciente y de que insistan en el apoyo y formación para reducir las tasas de error (Brown y Edelmann, 2000; Andersson et al, 2005; Ferguson y Day, 2007; Saintsing et al, 2011; Cleary et al, 2013).

La responsabilidad es un estresor importante que sufre una enfermera durante su proceso de transición. Esto, a la larga, afecta a un buen número de áreas de trabajo como la organización en el trabajo, la habilidad para la prioridad en determinados cuidados, tiempos de actuación, administración de medicación o en la toma de cualquier decisión. Es evidente que los altos niveles de ansiedad que sufren las nuevas enfermeras se relacionan con la responsabilidad en el desempeño de su rol (Clark y Holmes, 2007; Higgins et al, 2007; O'Shea y Kelly, 2007).

El hecho de considerar a las enfermeras como personal fácilmente intercambiable, es algo que se ha puesto en entredicho. Comenzar la carrera profesional en España no es algo fácil. Las enfermeras recién egresadas tienen contratos que cuestionan los mínimos de dignidad, esto pues se traduce en altos niveles de precariedad laboral y por tanto en inestabilidad laboral. En las organizaciones sanitarias se prioriza la política económica a corto plazo que la calidad. Las nuevas enfermeras que se incorporan a unidades de hospitalización, desconocidas para ellas, sólo cuentan con los conocimientos que adquieren durante los períodos de prácticas a lo largo de su formación. Las prácticas clínicas dan una visión general de la profesión, de ahí que los nuevos profesionales de enfermería puedan presentar dificultades para asumir pacientes con total responsabilidad. Durante los primeros años de vida profesional y laboral, las enfermeras, por lo general, trabajan cubriendo puestos en períodos de vacaciones o bajas, siendo contratos cortos. El desconocimiento de una nueva unidad de trabajo, de los protocolos a seguir, de las medicaciones a administrar, de los cuidados a realizar y las patologías a tratar genera inseguridad en las nuevas enfermeras (Benner, 1987:203; Ramió, 2005; Blanco García, 2012).

\section{Objetivo}

Profundizar en las vivencias de las enfermeras egresadas de la Universidad de La Laguna entre 2009 y 2014 con respecto a la responsabilidad, la inseguridad y el temor al error en el contexto laboral de Tenerife (España).

\section{METODOLOGÍA}

Se plantea un estudio cualitativo con un enfoque fenomenológico hermenéutico, mediante el análisis del discurso. Se toma como población de estudio los egresados titulados en enfermería entre los años 2009 y 2014 de la Universidad de La Laguna, de las dos escuelas universitarias de enfermería que están presentes en la isla de Tenerife: la Escuela Universitaria de Enfermería y Fisioterapia y la Escuela adscrita Ntra. Señora La Candelaria. Se toma una muestra aleatoria y por conveniencia de 19 participantes sin tener en cuenta ni la edad ni el sexo, habiendo participado 17 mujeres y 2 hombres (Tabla I). La recogida de datos se lleva a cabo con una sencilla entrevista: ¿En qué aspectos 
te sigues sintiendo insegura?; ¿Qué supone para ti cometer un posible error? A partir de la recogida de datos se realiza una selección, segmentación, codificación y categorización de las narraciones obtenidas. 16 entrevistas se realizan de forma oral y 4 por escrito.

\section{RESULTADOS}

\section{Categoría hacer}

Se establece una principal categoría denominada "hacer" que engloba todas las reflexiones acerca de la actuación de la enfermera durante el proceso de convertirse en profesional. Hace referencia a la ejecución del trabajo una vez la enfermera ya se encuentra dentro del contexto de trabajo donde ha de proceder a realizar diferentes técnicas y procedimientos al paciente. Estas técnicas y procedimientos entrañan ciertos riesgos para el usuario si no se realiza una praxis correcta y segura. Una actuación correcta y el temor al error están presentes en la enfermera una vez se encuentra trabajando en el contexto profesional. De hecho, cometer un error es considerado por las participantes de este estudio como un problema moral, un "cargo de conciencia", una vivencia emocional de impotencia y rabia, además de estresante, porque saben que tratan con personas y no con artículos materiales. Así pues, las enfermeras son conscientes de que cometer un error de actuación puede conllevar, incluso, a provocar la muerte en un paciente.

\section{Tabla I}

Población de estudiantes egresados de enfermería de las escuelas universitarias de enfermería en la isla de Tenerife

\begin{tabular}{|l|c|c|c|c|c|c|}
\hline & $\mathbf{2 0 0 9}$ & $\mathbf{2 0 1 0}$ & $\mathbf{2 0 1 1}$ & $\mathbf{2 0 1 2}$ & $\mathbf{2 0 1 3}$ & $\mathbf{2 0 1 4}$ \\
\hline $\begin{array}{l}\text { Diplomados en Enfermería. } \\
\text { ULL. Tenerife }\end{array}$ & 100 & 107 & 111 & 16 & 3 & - \\
\hline $\begin{array}{l}\text { Diplomados en Enfermería. } \\
\text { Ntra. Sra. de Candelaria }\end{array}$ & 59 & 62 & 74 & 4 & - & - \\
\hline $\begin{array}{l}\text { Graduados en Enfermería. } \\
\text { ULL. Tenerife }\end{array}$ & - & - & - & - & 82 & 98 \\
\hline Graduados en Enfermería. & - & - & - & - & 53 & 59 \\
Ntra. Sra. Candelaria & & & & & & \\
\hline
\end{tabular}

Fuente: Universidad de la Laguna. Elaboración propia. 
Las participantes manifiestan preocupación ante la realización de un procedimiento y equivocarse al realizarlo, siendo la principal preocupación cometer un error en la administración de fármacos, al ser el procedimiento que más entraña riesgos. Los errores además se pueden cometer cuando existe una sobrecarga de trabajo y las enfermeras han de realizar el procedimiento de la administración de fármacos de forma más rápida sin mucho tiempo para realizar comprobaciones de fármacos, paciente, vía de administración y hora de administración.

Existen diferentes tipos de errores según la gravedad y consecuencias que hayan podido tener en el paciente que atienden. Las profesionales consideran importante saber reconocer los errores y aprender de los mismos además de saber actuar con prudencia.

"Los primeros temores eran de equivocarme. Cometer algún error con algún paciente. Esos fueron mis miedos." (Participante 1)

"Matar a alguien. Cometer un error para mí supone mucho." (Participante 2)

"Las cargas laborales son una locura. Pueden hacer que cometas errores. Te pueden faltar cosas." (Participante 3)

"Terminé con tanta carga de trabajo que mi principal temor era equivocarme con la medicación. Eso era un pánico, que a veces llegas a tu casa dices ;Ay dios habré hecho esto bien, no registré lo otro!" (Participante 4)

"Pues depende del error que sea. No es lo mismo equivocarte al administrar medicación, al hacer una cura o que te equivoques en el tema del papeleo." (Participante 5)

"Para mí cometer un error depende del tipo de error. No me gustaría cometer un error vital, es decir, procuro trabajar en el sentido de no cometer errores. Si me veo insegura en algo no lo hago. Yo sí veo que eso puede repercutir en la vida del paciente reconozco mi capacidad para llevar a cabo esa situación y delego en otros profesionales o me dejo asesorar por otros profesionales en ese sentido. Siempre tiro del médico o de otros compañeros enfermeros. No soy imprudente la verdad." (Participante 6)

A partir de esta categoría principal se desarrollan las siguientes subcategorías, a continuación:

\section{Cometer un error}

Cometer un error para una enfermera supone un cargo de conciencia y es uno de los principales temores que manifiestan las enfermeras cuando realizan un procedimiento al paciente: hay temor a que al haber realizado una determinada actuación, se haga daño al paciente o se le perjudique en su mejoría.

"Un cargo de conciencia en el sentido de que has cometido un error. Me afecta porque he metido la pata, no quería hacerlo no es que me haya pasado $y$ después sabes que te van a decir algo. pero que no tengo tanto miedo porque lo primero que diría es que yo intenté hacerlo bien como soy responsable $y$ hago las cosa bien en ese aspecto no tengo tanto temor." (Participante 7)

"Error pues que haga algo mal y que el paciente sufra un daño de cualquier tipo sabes que te equivoques en una cura que el paciente este mucho más tiempo con esa cura o que o eso en una medicación que pase que gente vaya para atrás en su tratamiento en vez de que avance pues eso." (Participante 8) 


\section{Diferentes errores}

Existen diferentes errores en el día a día en el trabajo en enfermería que van de poco importantes a graves. La gravedad del error se centra principalmente si las consecuencias de dicho error perjudican seriamente la evolución o integridad del paciente y de si el error se puede solventar sin complicaciones. Es natural cometer errores: existen errores importantes que pueden perjudicar al paciente como es la administración de un fármaco de forma errónea en su dosis o en la vía de administración del mismo, o por el contrario cometer errores mucho menos graves como por ejemplo en las tareas administrativas.

"Está claro que depende del error. Todos tenemos fallos tontos y que no hacen daño a nadie. Para mí cometer un error me hace sentir muy pero que muy mal. Yo soy muy perfeccionista $y$ autoexigente por lo que no me gusta equivocarme en general pero ya si hablamos de aspectos que afectan a la salud del paciente pues más me angustio cuando me equivoco. De esto sobre todo al principio, ya no tanto porque yo misma digo "bueno en este caso, este fallo no le hace daño a nadie y más de uno se ha equivocado aquí." (Participante 9)

"En esta profesión cometer un error es algo bastante grave dependiendo de qué tipo de error se pueda cometer tratar pero cometer un error para mi es algo bastante grave es un poco no sé cómo explicártelo dependiendo del error si es un error que bueno lo solucionas fácilmente y otro que sean más graves entonces es algo muy importante no cometer errores en esta profesión." (Participante 10)
"Imagino yo que todos en algún momento de su vida han podido cometer un error. Depende del tipo de error: desde dar a un paciente un nolotil envés de un paracetamol o hasta el punto de darle una medicación al que era alérgico a esa medicación." (Participante 4)

"Depende de la gravedad del error pero cualquier error que cometas te hace sentirte muy mal te vas para casa con el rollo en la cabeza, es estresante cualquier error que cometas ya sea mínimo como olvidarte de una medicación que no importa que no le pusieras un antibiótico. Pero te vas para tu casa pensando me olvidé poner el antibiótico. O ya sea que te pases con la insulina $y$ le dé un bajón de azúcar y todo se solucione pero cualquier cosa de esas te llevas el rollo a la cabeza y estas un par de días con la matraquilla. Sabes que estás a la orden del día para cometer un error." (Participante 3)

\section{Errores en la medicación}

A continuación se abarcan los discursos de las participantes en torno a lo que supone y significa para ellas el hecho de cometer un error al administrar fármacos. Para la enfermera la administración de fármacos es un procedimiento que le inspira temor porque puede cometer un posible error en la preparación y administración de los mismos. Es una técnica que entraña riesgos y la enfermera ha de estar centrada y trabajar con seguridad. El temor a cometer un error en la administración de fármacos siempre está presente en las enfermeras, ya que si cometen un error en el fármaco a administrar, o administran un fármaco a un paciente por error o confusión, conlleva 
consecuencias o complicaciones graves en el paciente.

"A mí lo que más miedo me daba era cometer un error con la medicación. Era lo que más miedo me daba. Donde yo trabajé al principio preparábamos la medicación porque no había farmacia, entonces tenías que estar muy pendiente, porque aparte de la posible sanción, lo que más miedo me daba era equivocarme de medicación y darle algo a un usuario que fuera alérgico ya que hay mucha personas mayores con alergias". (Participante 11)

"No es lo mismo que yo a una úlcera le mande plata, le mande otras cosa que tampoco le va a pasar nada porque la cura se la haces cada tres días. Pero tú imagínate mandarle una medicación a un paciente que te ha dicho veinte mil veces que es alérgico a la penicilina y le mandes una penicilina. Eso para mí es un error, y gordo. Para mí sería algo con lo que pasaríamos a palabras mayores." (Participante 1.)

"Me miraba las cosas muchas veces antes de ponérselas al paciente. Aún hoy lo hago y voy a hacer un año de carrera. Pero sobre todo eso: yo en farmacológica no me encontraba preparada para nada. Lo demás más o menos pero cuando llevas medicación que sabes que el paciente puede tener peligro o causarle un daño, pues sí que miraba mucho." (Participante 12)

\section{Errores por sobrecarga de trabajo}

En este caso se observa, en los discursos de las siguientes enfermeras, que las situaciones estresantes y las cargas de trabajo hacen que cometan errores. La sobrecarga laboral conlleva trabajar más rápido y con más prisa. La enfermera se siente más estresada y no puede revisar meticulosamente y con serenidad todos los pasos a realizar cada vez que realiza un procedimiento, una técnica o la administración de un fármaco al paciente.

"Hay todo tipo de errores. Intentas hacerlo de la mejor manera posible intentando que no cometas ningún error pero las cargas de trabajo, el estar muy estresado...Con esto de las prisas seguro se nos escapa algún error." (Participante 4)

"Está claro que en una profesión donde hay mucho trabajo, sobrecarga, estrés, es imposible no equivocarse con algo, lo que pasa que muchos lo camuflan (porque lo he visto hacer) y eso sí que es peligroso." (Participante 9)

"En mi caso, aún no ha habido ningún error que deba mencionar. Los que he tenido han sido pequeños $y$ porque me he visto en ciertas situaciones estresantes que han desencadenado a ese error. En esos casos, he estado más pendiente $y$ he buscado ciertas técnicas que me facilitan el trabajo y evitar los errores." (Participante 13)

\section{Provocar la muerte}

Las participantes manifiestan lo conscientes que son trabajando como enfermeras y que un error puede tener consecuencias tan graves como la muerte de un paciente. Cometer un error para la enfermera supone un hecho que tiene una gran carga moral y de conciencia. Provocar una muerte por un error en la actuación o proceder de la enfermera, es un hecho muy grave. Muchas veces con la rutina del día a día y las sobrecargas laborales, los profesionales no son lo suficientemente conscientes de que un error puede llegar a 
tener consecuencias tan graves como puede ser provocar una muerte.

"A veces no somos conscientes o el sistema de trabajo que vamos rapidísimo no somos conscientes que cualquier fallito puede ocasionar la muerte a una persona." (Participante 14)

"El sistema tampoco ayuda y porque te das cuenta de que te metes en un mundo que es realmente importante porque estás trabajando con personas $y$ te das cuenta de que en realidad cualquier error puede provocar una muerte." (Participante 15)

\section{Reconocer los errores y aprender de los mismos}

Las participantes refieren que es importante que si se comete un error y se dan cuenta de que lo han cometido, deben tratar de solucionarlo lo antes posible. Un error es no comunicar que se ha cometido el error aún a sabiendas de que se ha cometido y puede tener consecuencias graves en el paciente. Es importante reconocer los errores cometidos, reflexionar sobre lo que ha sucedido, solventar el error, guardar la calma y aprender de los errores como una experiencia más de aprendizaje para que no vuelva a suceder.

"Yo creo que hay errores de muchos tipos. Depende de los errores. Lo más importante a la hora de cometer un error, es cuando lo cometes no callártelo porque eso sí no es un error, es una cosa súper grave. Si encima te lo callas como muchas veces pasa en los hospitales, tiene consecuencias para el paciente: eso es muy grave. Para mí lo importante es darte cuenta. Si lo cometes, pues intentar poner de tu parte para que eso no vaya a más." (Participante 15)

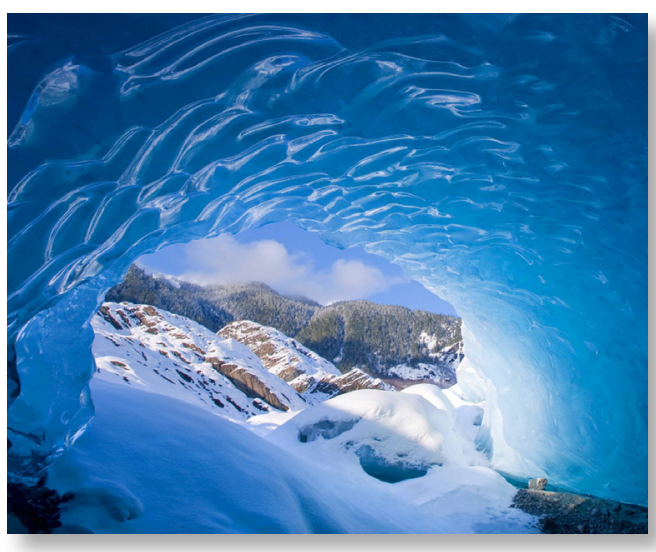

"En cualquier caso siempre intento mantener la calma, y aprender de los errores cometidos." (Participante 16)

"Sí es verdad que hay veces que los cometes y no sabes cómo. No te queda más remedio que tratar de solventarlo como puedas." (Participante 17)

\section{Actuar con prudencia}

Esta subcategoría se hace presente en los fragmentos analizados de las entrevistas donde se reflexiona sobre la importancia de la misma a la hora de trabajar siendo enfermera para no cometer errores, especialmente los graves. Son importantes los protocolos de actuación: con el desarrollo de los mismos se evita cometer muchos errores. Las enfermeras sienten que es importante dejarse asesorar, consultar dudas y trabajar de forma seria, meticulosa y ordenada. Actuar con prudencia es una virtud importante en el desempeño cotidiano del hacer enfermero.

"Para mí cometer un error comprende varios aspectos, uno de ellos si pone la vida o la integridad de un paciente en riesgo. Creo que depende de algún aspecto que se puede mejorar en la práctica: en el procedimiento, en el protocolo. No me preocupa si voy a cometer un error, no voy pensando en 
ello. Pero sí me preocuparía si pasara. Todo el mundo ha cometido errores en medicación y quien diga que no está equivocado. Eso falla en la forma segura, en los protocolos." (Participante 18)

"Para mí cometer un error es muchísimo, no me gusta nada. Sé que puede pasar y que puede pasar más veces. Yo todo lo que no sé, siempre pregunto." (Participante 19)

"Al haber estado en urgencias tienes un abanico amplio, tienes recursos para tratar todo tipo de pacientes pero siempre se te quedan cosas nuevas.... Ya en cuanto a seguridad del paciente... Aunque ya no te de miedo nada, el temor a cometer un error no lo podemos perder. Siempre hay que tener ese punto de precaución. Siempre tengo que revisar todo, tengo que trabajar metódicamente. Yo creo que nunca podemos relajarnos en ese aspecto de la profesión porque depende la vida del paciente." (Participante 19)

"Por eso creo que el enfermero tiene que ser un poco meticuloso, organizado y nunca confiarse porque cualquier error..." (Participante 14)

\section{DISCUSIÓN Y CONCLUSIONES}

Esta principal categoría que hemos desarrollado, en base al análisis de los discursos de las enfermeras, se construye sobre la actuación de la enfermera dentro del contexto laboral: los actos y procedimientos, la reflexión sobre los errores, las consecuencias de los errores y los sentimientos y reflexiones relacionados con ellos.

Los significados de las vivencias están centrados en cometer un posible error y en sus consecuencias. El error tiene mucho valor para la enfermera por las posibles consecuencias que pueda tener en el paciente: su empeoramiento o incluso provocar la muerte. De hecho es uno de los mayores temores que presentan nuestras enfermeras al comenzar a trabajar como profesionales. La consciencia del error supone un concepto importante en la nueva enfermera al entrar en el contexto laboral ya que saben que trabajan con personas. Por ello temen las prisas, el estrés, la acumulación de trabajo que requiere celeridad, las necesidades $\mathrm{y}$ demandas de pacientes en corto tiempo con escaso personal ya que pueden propiciar en errores que en no pocas ocasiones producen frustración y rabia en la enfermera.

El error que más preocupa a las nuevas enfermeras está relacionado con la administración de fármacos. Es el procedimiento a que más importancia dan las entrevistadas a la hora de realizarlo mal: fármaco incorrecto, dosis incorrecta, paciente incorrecto o farmacología compleja como la que se utiliza en las unidades de críticos. El significado dado al error es gradual, las enfermeras diferencian de entre errores más a menos graves, dependiendo del tipo de error puede tener mayor o menor temor para la enfermera: existen errores sin importancia y otros de mayor importancia si está en juego la integridad del paciente. En los discursos recogidos se observa que los errores graves están relacionados con el error en la administración de fármacos. En la profesión y en su día a día los errores pueden ser algo habitual, pero lo importante es darse cuenta del error, aprender del mismo y solucionarlo. Dado que los errores más graves están centrados en la administración de fármacos, en nuestro trabajo se propone mejorar los protocolos de actuación y la preparación en farmacología. Al reflexionar sobre los errores, 


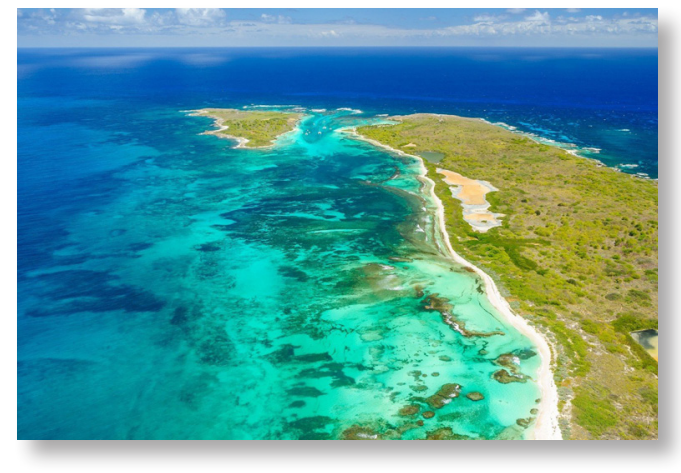

las enfermeras destacan la importancia de actuar con prudencia, para evitar los graves. Consultar las dudas, pedir ayuda, trabajar con rigor, son algunas de las formas de actuación para evitar los mismos.

En el modelo de transición de Shoessler y Waldo (2006), en la etapa ending que abarca los tres primeros meses de trabajo es donde la enfermera aprende a realizar tareas y procedimientos, el primer error es uno de los acontecimientos que marca a la enfermera en esta etapa como profesional. Observamos por tanto que coincidimos con estos autores en que el error es vivido de suma importancia para la nueva enfermera y sus discursos coinciden con el de los autores Saintsing et al, (2011). También coincidimos con dichos autores en la necesidad de la formación, en el apoyo a las enfermeras noveles para reducir sus tasas de error y así mejorar la seguridad del paciente. La inestabilidad laboral y no tener una ubicación fija genera inseguridad en la administración con seguridad de fármacos en las diferentes unidades (Blanco García, 2012) lo cual ocasiona incertidumbre y facilita cometer un posible error.

La inseguridad está relacionada con el contexto laboral (por estar sola en una unidad de trabajo y/o por comenzar en una unidad desconocida: rotación de servicios), por el temor al error, especialmente en la preparación y administración de fármacos.

\section{BIBLIOGRAFÍA}

- Andersson, N., Cederfäll, C., \& Klang, B. (2005). The novice general nurse's view of working in a paediatric setting: a Swedish experience. Nurse education in practice, 5(4), 191-197.

- Benner, P. (1987). Práctica progresiva en enfermería: Manual de comportamiento profesional. (1a. Ed.). Barcelona: Ediciones Grijalbo, SA.

- Blanco García, B. (2012). Los niveles de estrés de los estudiantes de enfermería: Programas de ayuda y acogida para los nuevos profesionales de enfermería. (Trabajo fin de máster). Oviedo: Universidad de Oviedo.

- Brown, H., \& Edelmann, R. (2000). Project 2000: a study of expected and experienced stressors and support reported by students and qualified nurses. Journal of advanced nursing, 31(4), 857-864.

- Clark, T., \& Holmes, S. (2007). Fit for practice? An exploration of the development of newly qualified nurses using focus groups. International journal of nursing studies, 44(7), 1210-1220.

- Cleary, M., Horsfall, J., Jackson, D., Muthulakshmi, P., \& Hunt, G. E. (2013). Recent graduate nurse views of nursing, work and leadership. Journal of clinical nursing. 22(19-20):2904-11. doi: 10.1111/jocn.12230.

- Cleary, M., Horsfall, J., Muthulakshmi, P., \& Jackson, D. (2013). A good day in nursing: Views of recent Singaporean graduates. Contemporary nurse, 45(1), 126-133.

- Ferguson, L. M., \& Day, R. A. (2007). Challenges for new nurses in evidence-based practice. Journal of Nursing Management, 15(1), 107-113.

- Higgins, G., Spencer, R. L.,\& Kane, R. (2010). A systematic review of the experiences and perceptions of the newly qualified nurse in the United Kingdom. Nurse Education Today, 30(6), 499-508.

- O'Shea, M., \& Kelly, B. (2007). The lived experiences of newly qualified nurses on clinical placement during the first six months following registration in the Republic of Ireland. Journal of Clinical Nursing, 16(8), 1534-1542.

- Ramió, A. (2005). Valores y actitudes profesionales. Estudio de la práctica profesional enfermera en Cataluña (Tesis Doctoral). Departamento de Sociología y Análisis de las Organizaciones. Facultad de Ciencias Económicas y Empresariales. Universidad de Barcelona.

- Saintsing, D., Gibson, L. M., \& Pennington, A. W. (2011). The novice nurse and clinical decision-making: how to avoid errors. Journal of Nursing Management, 19(3), 354359.

- Schoessler, M., \& Waldo, M. (2006). The first 18 months in practice: a developmental transition model for the newly graduated nurse. Journal for Nurses in Professional Development, 22(2), 47-52 